\title{
A Deterministic Mathematical Model for Direct and Indirect Transmission Dynamics of Typhoid Fever
}

\author{
Stephen Edward \\ Department of Mathematics, University of Dodoma, Dodoma, Tanzania \\ Email: stephenmwaihuti@yahoo.com
}

How to cite this paper: Edward, S. (2017) A Deterministic Mathematical Model for Direct and Indirect Transmission Dynamics of Typhoid Fever. Open Access Library Journal, 4: e3493.

https://doi.org/10.4236/oalib.1103493

Received: March 4, 2017

Accepted: April 30, 2017

Published: May 3, 2017

Copyright (c) 2017 by author and Open Access Library Inc.

This work is licensed under the Creative Commons Attribution International License (CC BY 4.0).

http://creativecommons.org/licenses/by/4.0/

\section{c) (†) Open Access}

\begin{abstract}
Improvements in sanitation and the provision of clean drinking water led to the elimination of typhoid fever from developed countries in the beginning of the 20th century. However, Salmonella typhi and paratyphi remain a major source of morbidity and mortality in many developing countries today. The dynamics of typhoid transmission are poorly understood. In this study, we develop a novel mathematical model that captures the role of both human to human interaction and human to environment interaction in the transmission dynamics of typhoid fever. Our results have shown the feasible impact of different methods of typhoid control, including vaccination, improved treatment strategies, and investment in clean water and sanitation.
\end{abstract}

\section{Subject Areas}

Mathematical Modeling

\section{Keywords}

Modeling, Sanitation, Treatment, Vaccination, Epidemiology, Typhoid

\section{Introduction}

Typhoid fever is a communicable disease, found only in man and occurs due to systemic infection mainly by Salmonella typhi organism [1]. The disease is endemic in many developing countries and despite recent progress in water and sanitation coverage, it remains a substantial public health problem. Globally, it is estimated that typhoid causes over 16 million cases of illness each year, resulting in over 600,000 deaths [2]. Typhoid has a long storied history as a public health scourge. Salmonella enterica serovar Typhi $(S$. Typhi) is a human restricted bacterial pathogen transmitted via fecal contamination of food and water [3]. While 
improvements in water and sanitation led to the elimination of typhoid from most developed countries during the twentieth century, the global burden of typhoid fever has recently been estimated to be between 13.5 and 26.9 million episodes and 190,000 to 216,000 deaths annually [4]. In many developing nations, the public health goals that can help prevent and control the spread of typhoid fever disease through safe drinking water, improved sanitation and adequate medical care may be difficult to achieve. Health education is paramount to raise public awareness and induce behavior change [5]. Several mathematical models have been developed to explain the dynamics of typhoid including [2] [6]-[12], but none has incorporated both direct and indirect transmission dynamics in typhoid fever model.

Our main objective in the present paper is to develop an $S I I c R-B$ (susceptible, symptomatic infectious, asymptomatic infectious, recovered, bacteria concentration) model of typhoid fever with vaccination, treatment and water sanitation as control strategies that has not been investigated in prior studies. Our major assumption is existence of both direct transmission of typhoid from infected individuals to susceptible and indirect transmission of bacteria from the environment to the susceptible, the other assumptions; all susceptible individuals are equally likely to be infected by infectious individuals in case of contact, and we also assume direct transmission of typhoid from infected to susceptible individuals and that there is a constant recruitment rate to the susceptible population. Furthermore, we assume that the rate of transmission for carriers is greater than that of symptomatic infectious individuals.

\section{Model Formulation}

We first develop a more realistic model for typhoid. The model subdivides the human population of interest into four compartments: susceptible humans $(S)$, infected humans $(I)$, carrier humans $\left(I_{c}\right)$, and recovered humans $(R)$. Previous models of typhoid dynamics [2] [6]-[12], assume direct transmission of typhoid from infected individuals to susceptible individuals. However, typhoid is largely contracted from environmental bacteria through contaminated water and/or food and drinks [13] [14], and transmission of typhoid through direct person-to-person contact, if any, is negligible [15]. To incorporate this real biological phenomenon, we consider an additional compartment, $B$, which represents bacteria in the environment. We assume that susceptible individuals get infected with typhoid at a rate proportional to the susceptible population, $S$, and the environmental bacteria concentration, $B$, at a constant rate $\tau$.

Individuals in the class, $I$, can recover from typhoid at the rate, $\eta_{1}$. The carrier individuals can also either progress to carrier class, $I_{c}$, at rate $\alpha$ or recover from typhoid, but with a significantly slow rate, $\eta_{2}$. Infected individuals in both infectious state and carrier state excrete bacteria into the environment. However, the rate of excretion by the infectious group, $\epsilon_{1}$, is significantly higher than that by the carrier group, $\epsilon_{2}$. Note that despite low excretion of bacteria by the carrier group, because of its extremely long duration without showing any sickness 
the carrier group plays an important role on infection dynamics of typhoid. Growth curves of organisms are often described well with the 2 logistic models [16]-[21], so we assume that the bacteria in the environment grows according to a logistic growth rate and becomes non-infectious at a rate $\mu_{b} . r$ and $K$ represent per capita growth rate and carrying capacity, respectively, and $d_{1}, d_{2}$ denotes the typhoid induced mortality in Infectious and carriers individuals respectively. The constant recruitment rate into the susceptible human is represented by $\Lambda$, while the natural death rate of human is represented by $\mu_{h}$. The developed model can be expressed as the following differential equations detailed in section 2.1 .

\subsection{Model Equations}

From the assumptions, descriptions and the compartmental diagram in Figure 1 , we formulate the following system of differential equations.

$$
\begin{gathered}
\frac{\mathrm{d} S}{\mathrm{~d} t}=\Lambda+\phi R-\left(\lambda+\mu_{h}+\theta\right) S \\
\frac{\mathrm{d} I}{\mathrm{~d} t}=p \lambda S+\alpha I_{c}-\left(\mu_{h}+d_{1}+\eta_{1}+\epsilon_{1}\right) I \\
\frac{\mathrm{d} I_{c}}{\mathrm{~d} t}=(1-p) \lambda S-\left(\alpha+\mu_{h}+d_{2}+\eta_{2}+\epsilon_{2}\right) I_{c} \\
\frac{\mathrm{d} B}{\mathrm{~d} t}=r\left(1-\frac{B}{K}\right) B+\epsilon_{1} I+\epsilon_{2} I_{c}-\mu_{b} B \\
\frac{\mathrm{d} R}{\mathrm{~d} t}=\eta_{1} I+\eta_{2} I_{c}+\theta S-\left(\mu_{h}+\phi\right) R \\
\frac{\mathrm{d} N}{\mathrm{~d} t}=\Lambda-\mu_{h} N-d_{1} I-d_{2} I_{c}
\end{gathered}
$$

where

$$
\lambda=\beta I+\gamma I_{c}+\frac{\tau B}{K+B}
$$

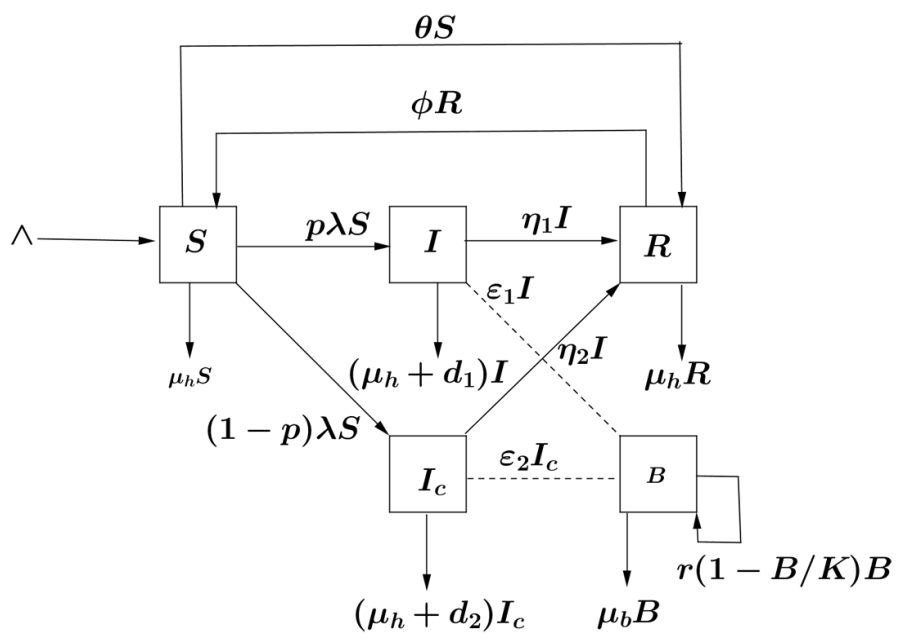

Figure 1. A compartmental diagram for a typhoid model with control strategies. 
Equation (1) describes the dynamics of susceptible in the community of size $N$. The death rate of the susceptible individuals is represented by, is the rate of recruitment into susceptible class, is the rate of exposure to contaminated food and water, is the probability of susceptible catching typhoid fever, is the susceptible and is the concentration of Salmonella typhi bacteria in food and water. Equations (2) and (3) describes the dynamics of infected people in the community, their number increases as susceptible become infected and decreases as the infected recovers or die from the disease or natural death. Measures to limit the spread of the disease, such as hygiene and total sanitation reduce the amount of Salmonella typhi bacteria in the environment. Equation (4) describes the dynamics of pathogenic Salmonella typhi bacteria in environment, comprising the contaminated food or water consumed by people and unhygienic handling of typhoid fever patients and their waste products. Equation (5) describes the dynamics of effect of treatment or lack of treatment to the population of infected people.

\subsection{Basic Properties of the Model}

\subsubsection{Positivity of Solutions}

We show that if the system starts with non-negative initial conditions $\left(S^{0}, I^{0}, I_{c}^{0}, R^{0}, B^{0}\right)$, the solutions/trajectories of (1)-(5) will remain non-negative for all $t \in(0, \infty)$. This is an ideal condition to check since the model monitors human population and the pathogen concentration in the aquatic environment. We thus have the following theorem: Theorem 1. Given that the initial conditions of the system (1)-(5) $\left(S^{0}>0, I^{0}>0, I_{c}^{0}>0, R^{0}>0, B^{0}>0\right)$, the resulting solutions $\left(S(t), I(t), I_{c}(t), R(t), B(t)\right)$ are all non-negative for all $t \in(0, \infty)$.

Proof. To show positivity of solution, it is enough to show that each of the tra-jectories of system (1)-(5) is non negative for all $t>0$. From Equation (1), the differential inequality describing the evolution of the susceptible population over time is given by

$$
\frac{\mathrm{d} S}{\mathrm{~d} t} \geq \Lambda-\left(\lambda+\mu_{h}+\theta\right) S
$$

The resulting differential inequality can be solved by separation of variables. Since at $t=0, S(0)=S^{0}$, then the complete solution to the differential inequality for the susceptible population is given by

$$
\begin{gathered}
S(t) \geq \frac{\Lambda}{\mu_{h}+\theta}+\left(S^{0}-\frac{\Lambda}{\mu_{h}+\theta}\right) \mathrm{e}^{-\left(\mu_{h}+\theta\right) t} \\
\lim _{n \rightarrow \infty} \inf S(t) \geq 0
\end{gathered}
$$

Using the same principle, the rest of the phase space variables as $t$ approaches infinity can be shown to satisfy

$$
\begin{gathered}
I(t) \geq I^{0} \mathrm{e}^{-\left(\mu_{h}+d_{1}+\eta_{1}+\epsilon_{1}\right) t}, \\
I_{c}(t) \geq I^{0} \mathrm{e}^{-\left(\mu_{h}+d_{2}+\eta_{2}+\epsilon_{2}\right) t}
\end{gathered}
$$




$$
R(t) \geq R_{0} \mathrm{e}^{-\left(\mu_{h}+\phi\right) t}
$$

From which the limit inf of the corresponding state variables can be shown to be non-negative. Using the equation describing the evolution of the pathogen concentration, we have a differential inequality given by

$$
\frac{\mathrm{d} B}{\mathrm{~d} t}+\left(\mu_{b}-r\right) B \geq-\frac{B^{2}}{K}
$$

Equation (9) is a Bernoulli type of equation. It is solved by substitution i.e. $B=y^{-1}$ to obtain

$$
B(t) \geq-\frac{\mathrm{e}^{-\left(\mu_{b}-r\right) t}}{A K\left(\mu_{b}-r\right)}
$$

\subsubsection{Boundedness of Solutions}

The model can be separated into two parts which include, the human population $T_{H}$ and the concentration of the pathogen in the aquatic environment $T_{B}$ such that $T_{H}=\left\{\left(S(t), I(t), I_{c}(t), R(t)\right) \in \mathbb{R}_{+}^{4}: S+I+I_{c}+R=N\right\}$ and $T_{B}=\left\{B(t) \in \mathbb{R}_{+}^{1}\right\}$ respectively. From Equation (1) the differential inequality of the susceptible population is given by

$$
\begin{gathered}
\frac{\mathrm{d} S}{\mathrm{~d} t}=\Lambda+\phi R-\left(\lambda+\mu_{h}+\theta\right) S \\
\frac{\mathrm{d} S}{\mathrm{~d} t}+\left(\mu_{h}+\theta\right) S \leq \Lambda+\phi R
\end{gathered}
$$

Using a suitable integrating factor, $I . F=\mathrm{e}^{-\mu t}$, the differential inequality (11) can be solved to obtain

$$
S(t) \leq \frac{\Lambda}{\mu_{h}}+\mathrm{e}^{-\mu t} \int_{0}^{t} \omega R(x) \mathrm{e}^{-\mu x} \mathrm{~d} x
$$

Following the theorem of differential inequality by Birkhoff and Rota [21] we obtain

$$
\lim _{t \rightarrow \infty} \operatorname{Sup} S(t) \leq \frac{\Lambda}{\mu_{h}}
$$

Therefore, the state variable describing the evolution of the susceptible population is less or equal to the ratio of the recruitment rate and the natural mortality rate. We note also that the total population is given as $N=S+I+I_{c}+R$. If we take the time derivative of $N$ i.e. $\frac{\mathrm{d} N}{\mathrm{~d} t}$ and substitute the Equations (1)-(4) into the resulting expression we obtain

$$
\frac{\mathrm{d} N}{\mathrm{~d} t}=\Lambda-\mu_{h} N-d_{1} I-d_{2} I_{c}
$$

The solution (12) can be obtained by separating variables and integrating both sides with respect to the corresponding variable. This result into

$$
\ln \left|\Lambda-\mu_{h} N\right| \geq-\mu_{h} t+c
$$

where $c$ is a constant of integration. If we exponentiate both sides and assume that the initial total population is $N^{0}$, the solution becomes 


$$
N \leq \frac{\Lambda}{\mu_{h}}-\left(\frac{\Lambda}{\mu_{h}}-N^{0}\right) \mathrm{e}^{-\mu_{h} t}
$$

therefore,

$$
\lim _{t \rightarrow \infty} \operatorname{Sup} N(t) \leq \frac{\Lambda}{\mu_{h}}
$$

Since $N$ is the sum of all state space variables, then each of the individual state variables is less or equal to $\frac{\Lambda}{\mu_{h}}$. Using Equation (5), we assume that the growth rate of the pathogen in linear at a constant rate $r$. We therefore obtain a differential inequality

$$
\begin{gathered}
\frac{\mathrm{d} B}{\mathrm{~d} t}+\left(\mu_{b}-r\right) B \leq \epsilon_{1} I+\epsilon_{2} I_{c} \\
\frac{\mathrm{d} B}{\mathrm{~d} t}+\left(\mu_{b}-r\right) B \leq\left(\epsilon_{1}+\epsilon_{2}\right) \frac{\Lambda}{\mu_{h}}
\end{gathered}
$$

The solution to this equation can be obtained by using a suitable integrating factor to obtain

$$
B(t) \leq \frac{\left(\epsilon_{1}+\epsilon_{2}\right) \Lambda}{\mu_{h}\left(\mu_{b}-r\right)}+A \mathrm{e}^{\left(\mu_{b}-r\right) t}
$$

therefore,

$$
\lim _{t \rightarrow \infty} \operatorname{Sup} B(t) \leq \frac{\left(\epsilon_{1}+\epsilon_{2}\right) \Lambda}{\mu_{h}\left(\mu_{b}-r\right)}
$$

The domain of biological significance of the system (1)-(5) is

$$
T=\left[S, I, I_{c}, R, B(t) \geq 0: S+I+I_{c}+R \leq \frac{\Lambda}{\mu_{h}}, B(t) \leq \frac{\left(\epsilon_{1}+\epsilon_{2}\right) \Lambda}{\mu_{h}\left(\mu_{b}-r\right)}\right] .
$$

The domain $T$ is positively invariant under the flow induced by the system (1)-(5). Therefore, the system (1)-(5) is biologically meaningful and it is feasible to analyze the model in the domain $T$.

\subsection{The Basic Reproduction Number, $\boldsymbol{R}_{0}$}

The basic reproduction number denoted by $R_{0}$ is the average number of secondary infections caused by an infectious individual during his or her entire period of infectiousness Diekmann et al. [22]. The basic reproduction number is an important non-dimensional quantity in epidemiology as it sets the threshold in the study of a disease both for predicting its outbreak and for evaluating its control strategies. Thus, whether a disease becomes persistent or dies out in a community depends on the value of the reproduction number, $R_{0}$. Furthermore, stability of equilibria can be analyzed using $R_{0}$. If $R_{0}<1$ it means that every infectious individual will cause less than one secondary infection and hence the disease will die out and when $R_{0}<1$, every infectious individual will cause more than one secondary infection and hence the disease will invade the population. A 
large number of $R_{0}$ may indicate the possibility of a major epidemic. For the case of a model with a single infected class, $R_{0}$ is simply the product of the infection rate and the mean duration of the infection. In more complicated epidemics we compute the basic reproduction number, $R_{0}$ using the next generation operator approach by Van den Driessche and Watmough [23]. We calculate the basic reproduction number by using the next generation operator method on the system Equation (1)-(5). The basic reproduction number is obtained by taking the largest (dominant) eigenvalue (spectral radius)

$$
F V^{-1}=\left[\frac{\partial \mathcal{F}_{i}\left(E_{0}\right)}{\partial x_{j}}\right]\left[\frac{\partial \mathcal{V}_{i}\left(E_{0}\right)}{\partial x_{j}}\right]^{-1}
$$

where $F_{i}$ is the rate of appearance of new infection in compartment $i, V_{i}$ is the transfer of infections from one compartment $i$ to another and $E^{0}$ is the disease-free equilibrium. From system equation of the system (1)-(4), we re-write the equations with infectious classes, $I, I_{c}$ and $B$. This leads to the system

$$
\begin{gathered}
\frac{\mathrm{d} I}{\mathrm{~d} t}=p \lambda S+\alpha I_{c}-\left(\mu_{h}+d_{1}+\eta_{1}+\epsilon_{1}\right) I \\
\frac{\mathrm{d} I_{c}}{\mathrm{~d} t}=(1-p) \lambda S-\left(\alpha+\mu_{h}+d_{2}+\eta_{2}+\epsilon_{2}\right) I_{c} \\
\frac{\mathrm{d} B}{\mathrm{~d} t}=r\left(1-\frac{B}{K}\right) B+\epsilon_{1} I+\epsilon_{2} I_{c}-\mu_{b} B
\end{gathered}
$$

Jacobian at diseases free point $\left(E_{0}\right)$ is computed and found to be

$$
J\left(E_{0}\right)=\left[\begin{array}{c}
\frac{p \tau B S}{K+B}+p \beta I S+p \gamma I_{c} S \\
\frac{(1-p) \tau B S}{K+B}+(1-p) \beta I S+(1-p) \gamma I_{c} S \\
0
\end{array}\right]
$$

from which we obtain:

$$
\begin{gathered}
\mathcal{F}_{i}=\left[\begin{array}{c}
\frac{p \tau B S}{K+B}+p \beta I S+p \gamma I_{c} S \\
\frac{(1-p) \tau B S}{K+B}+(1-p) \beta I S+(1-p) \gamma I_{c} S \\
0
\end{array}\right] \\
\mathcal{V}_{i}=\left[\begin{array}{c}
\left(\mu_{h}+d_{1}+\eta_{1}+\epsilon_{1}\right) I \\
\left(\mu_{h}+d_{2}+\eta_{2}+\epsilon_{2}\right) I_{c} \\
\mu_{b} B-\epsilon_{1} I \epsilon_{2} I_{c}+r\left(1-\frac{B}{K}\right) B
\end{array}\right]
\end{gathered}
$$

Partial differentiation of $F_{i}$ and $V_{i}$ with respect to $I, I_{c}$ and $B$ gives 


$$
\begin{gathered}
F=\left[\begin{array}{ccc}
p \beta S^{0} & p \gamma S^{0} & \frac{p \tau S^{0}}{K} \\
(1-p) \beta S^{0} & (1-p) \beta S^{0} & \frac{(1-p) \tau S^{0}}{K} \\
0 & 0 & 0
\end{array}\right] \\
V=\left[\begin{array}{ccc}
\left(\mu_{h}+d_{1}+\eta_{1}+\epsilon_{1}\right) & 0 & 0 \\
0 & \left(\mu_{h}+d_{2}+\eta_{2}+\epsilon_{2}\right) & 0 \\
-\epsilon_{1} & -\epsilon_{2} & \mu_{b}-r
\end{array}\right]
\end{gathered}
$$

$V^{-1}$ was computed and found to be:

$$
V^{-1}=\frac{1}{a_{1} a_{2}\left(\mu_{b}-r\right)}\left[\begin{array}{ccc}
a_{2}\left(\mu_{b}-r\right) & \alpha\left(\mu_{b}-r\right) & 0 \\
0 & a_{1}\left(\mu_{b}-r\right) & 0 \\
\epsilon_{1} a_{2} & a \epsilon_{2} & a_{1}
\end{array}\right]
$$

Lastly $F V^{-1}$ was calculated and results is:

$$
F V^{-1}=\frac{1}{a_{1} a_{2}\left(\mu_{b}-r\right)}\left(\begin{array}{ccc}
p \beta S^{0} a_{2}\left(u_{b}-r\right)+\frac{p \tau S^{0} \epsilon_{1} a_{2}}{K} & p \gamma a_{1} S^{0}\left(u_{b}-r\right)+\frac{p \tau S^{0} f}{K} & \frac{p a_{1} \tau S^{0}}{K} \\
(1-p) \beta S^{0} a_{2}\left(u_{b}-r\right)+\frac{(1-p) \tau S^{0} \epsilon_{1} a_{2}}{K} & (1-p) \gamma a_{1} S^{0}\left(u_{b}-r\right)+\frac{(1-p) a_{1} \tau S^{0} f}{K} & \frac{(1-p) a_{1} \tau S^{0}}{K} \\
0 & 0 & 0
\end{array}\right)
$$

The eigenvalues of $F V^{-1}$ was calculated as follows:

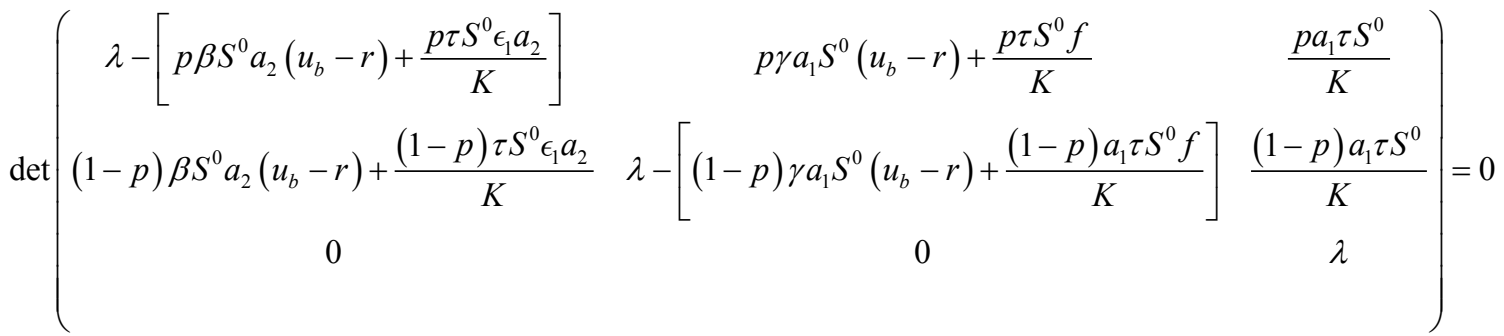

let

$$
\begin{gathered}
Z_{1}=p \beta S^{0} a_{2}\left(u_{b}-r\right)+\frac{p \tau S^{0} \epsilon_{1} a_{2}}{K} \\
Z_{2}=p \beta S^{0} \alpha\left(u_{b}-r\right)+p \gamma a_{1} S^{0}\left(u_{b}-r\right)+\frac{p \tau S^{0} f}{K} \\
Z_{3}=\frac{p \tau S^{0}}{K} \\
Z_{4}=(1-p) \beta S^{0} a_{2}\left(u_{b}-r\right)+\frac{(1-p) \tau S^{0} \epsilon_{1} a_{2}}{K} \\
Z_{5}=(1-p) \gamma a_{1} S^{0}\left(u_{b}-r\right)+\frac{(1-p) a_{1} \tau S^{0} f}{K} \\
Z_{6}=\frac{(1-p) a_{1} \tau S^{0}}{K}
\end{gathered}
$$


We get

$$
\begin{gathered}
\left|\begin{array}{ccc}
\lambda-Z_{1} & Z_{2} & Z_{3} \\
Z_{4} & \lambda-Z_{5} & Z_{6} \\
0 & 0 & \lambda
\end{array}\right|=0 \\
\lambda\left[\left(\lambda-Z_{5}\right)\left(\lambda-Z_{1}\right)-Z_{2} Z_{5}\right]=0 \\
\lambda\left(\lambda^{2}-\left(Z_{1}+Z_{5}\right) \lambda+\left(Z_{1} Z_{5}-Z_{2} Z_{4}\right)\right)=0
\end{gathered}
$$

it can be shown that $Z_{1} Z_{5}-Z_{2} Z_{4}=0$

$$
\begin{gathered}
\lambda^{2}\left(\lambda-\left(Z_{1}+Z_{5}\right)\right)=0 \\
\lambda=0,0, Z_{1}+Z_{5}
\end{gathered}
$$

thus the maximum eigenvalues is

$$
Z_{1}+Z_{5}
$$

which gives

$$
R_{e}=Z_{1}+Z_{5}
$$

therefore

$$
\begin{gathered}
R_{e}=p \beta S^{0} a_{2}\left(\mu_{b}-r\right)+a_{1}(1-p) \gamma S^{0}\left(\mu_{b}-r\right)+\frac{p \tau S^{0} \epsilon_{1} a_{2}}{K}+\frac{(1-p) \tau S^{0} a_{1} \epsilon_{2}}{K} \\
=\frac{S^{0}}{a_{1} a_{2}\left(\mu_{b}-r\right)}\left(\left(\mu_{b}-r\right)\left[p a_{2} \beta+a_{1}(1-p) \gamma\right]+\frac{\tau}{K}\left[a_{2} p \epsilon_{1}+(1-p) a_{1} \epsilon_{2}\right]\right) \\
=\frac{S^{0} p}{a_{1}\left(\mu_{b}-r\right)} \frac{\tau}{K}\left(\beta\left(\mu_{b}-r\right)+\frac{\tau \epsilon_{1}}{K}\right)+\frac{(1-p) S^{0}}{a_{2}\left(\mu_{b}-r\right)} \\
=\left(\frac{S^{0} p}{a_{1}} \beta+\frac{S^{0} p \tau}{a_{1}\left(\mu_{b}-r\right) K} \epsilon_{1}\right)+\left(\frac{(1-p) S^{0}}{a_{2}} \gamma+\frac{\tau}{K} \frac{(1-p) S^{0} \gamma}{a_{2}\left(\mu_{b}-r\right)} \epsilon_{2}\right)
\end{gathered}
$$

This can be written as

$$
R_{e}=\left(R_{01}+R_{03}\right)+\left(R_{02}+R_{04}\right)
$$

where

$$
\begin{gathered}
R_{01}=\left(\frac{S^{0} p}{a_{1}}\right) \\
R_{02}=\frac{S^{0}(1-p) \gamma}{a_{2}} \\
R_{03}=\left(\frac{S^{0} p \tau}{a_{1}\left(\mu_{b}-r\right) K}\right) \epsilon_{1} \\
R_{04}=\frac{S^{0}(1-p) \tau}{a_{2}\left(\mu_{b}-r\right) K} \epsilon_{2} \\
S^{0}=\frac{\left(\mu_{h}+\phi\right) \Lambda}{\mu_{h}\left(\mu_{h}+\theta+\phi\right)}
\end{gathered}
$$




$$
\begin{gathered}
a_{1}=\mu_{h}+d_{1}+\eta_{1}+\epsilon_{1} \\
a_{2}=\mu_{h}+d_{2}+\eta_{2}+\epsilon_{2}
\end{gathered}
$$

Thus $R_{e}$ is the effective reproduction number (basic reproduction number with controls). The terms $\frac{1}{a_{1}}$ and $\frac{1}{a_{2}}$ indicate the maximum time an individual is expected to stay in compartments $I$ and $I_{c}$ respectively. The reproduction number consists of four terms which characterize the contribution from the different pathways to new infections with typhoid.

\section{Results and Discussion}

In this study our objective is to model transmission dynamics of typhoid fever via the direct and indirect paths, we want to analyze what happens to the system when control measures like vaccination, treatment and water sanitation either effected or not. Furthermore we want to know the role of carriers and symptomatic individuals to this dynamical system. Various graphical representations have been generated with the help of MATLAB which will support our analytical results. Since, most of the parameters were not readily available; it was found convenient to pick from other sources and unavailable data were estimated. In order to perform simulations, baseline values of parameters from Table 1 presented before were used.

It can be seen from Figures 2(a)-(d) that sanitation, vaccination, treatment of both symptomatic and asymptomatically infected individuals respectively, are observed to reduce the severity of the disease if such parameters are increased.

\begin{tabular}{|c|c|c|c|}
\hline Parameter & Value & Description & Source \\
\hline$\Lambda$ & $10^{6}$ & Constant human recruitment rate & {$[10][13]$} \\
\hline$\mu_{b}$ & $0.4 /$ year & Mortality rate for bacteria, including phage degradation & Estimated \\
\hline$\mu_{h}$ & $0.167 /$ year & Natural human mortality rate & Estimated \\
\hline$d_{1}$ & $0.15 /$ year & Disease induced death rate & {$[2]$} \\
\hline$d_{2}$ & $0.6 /$ year & Disease induced death rate & Estimated \\
\hline$\beta$ & $0.02 /$ year & Effective contact rate between individuals (contact sufficient) & Estimated \\
\hline$\tau$ & $0.7 /$ year & Per capita contact rate for humans and contaminated water & Estimated \\
\hline$\eta_{1}$ & $0.04 /$ year & Recovery rate of infectious humans & {$[4]$} \\
\hline$\eta_{2}$ & $0.05 /$ year & Recovery rate of infectious humans & Estimated \\
\hline$\epsilon_{1}$ & $0.5 /$ year & Bacteria shed rate into the water supply by infectious human & Estimated \\
\hline$\epsilon_{2}$ & $0.4 /$ year & Bacteria shed rate into the water supply by infectious human & Estimated \\
\hline$\phi$ & $0.33 /$ year & Per capita rate at which recovered humans are susceptible & Estimated \\
\hline$\theta$ & $0.4 /$ year & Per capita rate at which susceptible humans are vaccinated & {$[10]$} \\
\hline$r$ & $0.01 /$ year & (Maximum) per capita growth rate for S. typhi bacteria & {$[10]$} \\
\hline$K$ & $100 /$ year & Carrying capacity for S. typhi & Estimated \\
\hline$p$ & $0.8 /$ year & Proportion of infected individuals who are symptomatic & Estimated \\
\hline
\end{tabular}

Table 1. Parameters and their description. 


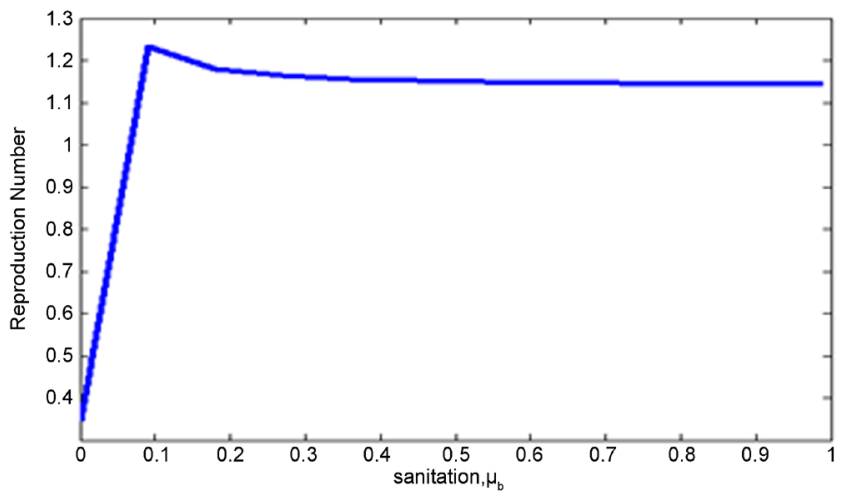

(a)

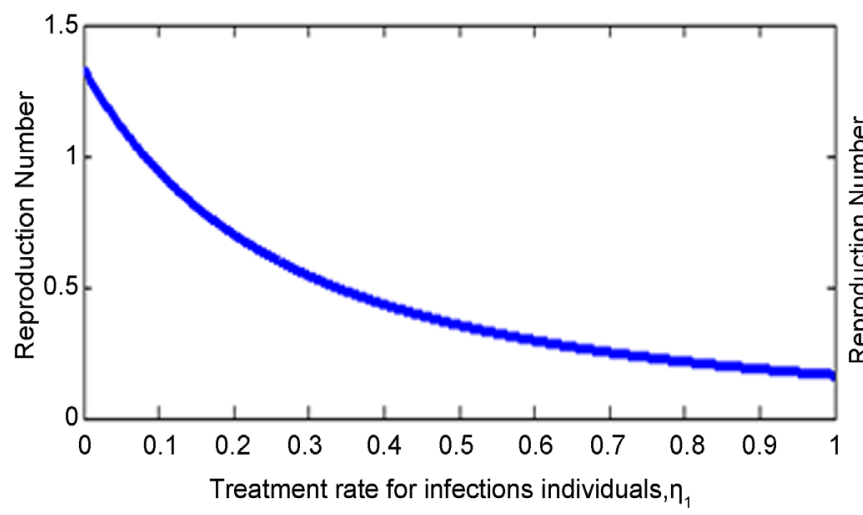

(c)

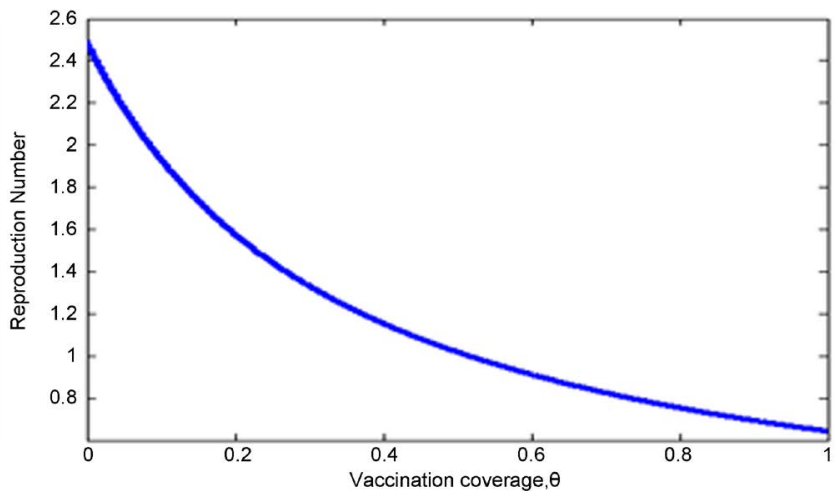

(b)

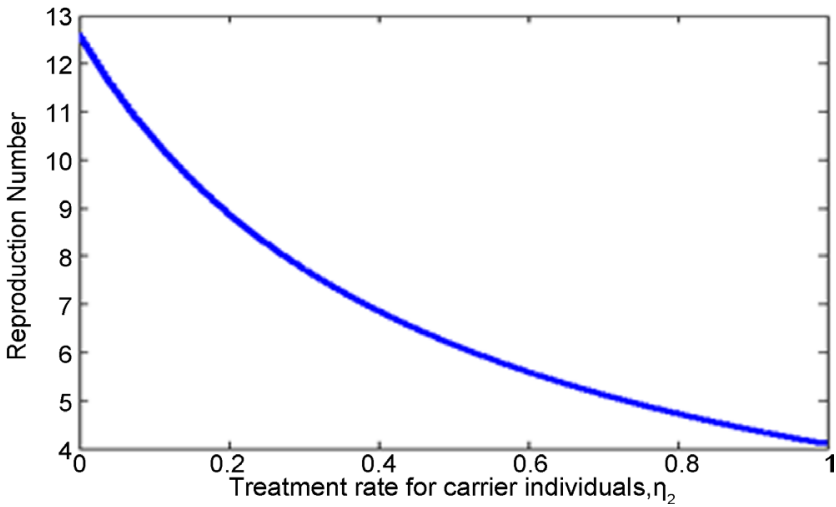

(d)

Figure 2. (a)shows the effect of sanitation effort to the effective reproduction number, (b) shows the effect of variation of vaccination coverage on the effective reproduction number, (c) shows effect of variation of therapeutic treatment of symptomatic infectious individuals on the effective reproduction number and (d) shows effect of variation of therapeutic treatment of asymptomatic (carrier) infectious individuals on the effective reproduction number.

Recovery of infected individuals has a twofold benefit in the fight against the infection; 1) it leads to reduction the likelihood getting new infections through direct person to person transmission, and 2) a negligible amount of the pathogen would be shed into the aquatic reservoir greatly reducing the infection risk, most especially for the community that may have direct contact with a potentially contaminated water source. In addition, recovering individuals acquire some immunity to the disease which only wanes over a reasonable period of time hence reducing the susceptible. On the other hand, it can be seen from Figures $3(a)-(e)$ that increased discharge of fresh bacteria into the aquatic environment by either the symptomatic or asymptomatic individuals, increased probability of contracting bacteria from the environment. Likewise, high personal contact rate with either the symptomatic or asymptomatic individuals increase the risk of contracting typhoid fever. The worst case scenario in case of epidemic outbreak may be experienced when the level of hygiene is poor; maximizing person-toperson contact rate and when there is no accesses to clean water; which maximizes disease transmission through contact with the contaminated reservoir. If immunization of the susceptible population alone does not bring about typhoid elimination, then measures to reduce per case or per carrier infectivity, such as improved sanitation or hand washing with soap, might be considered instead 


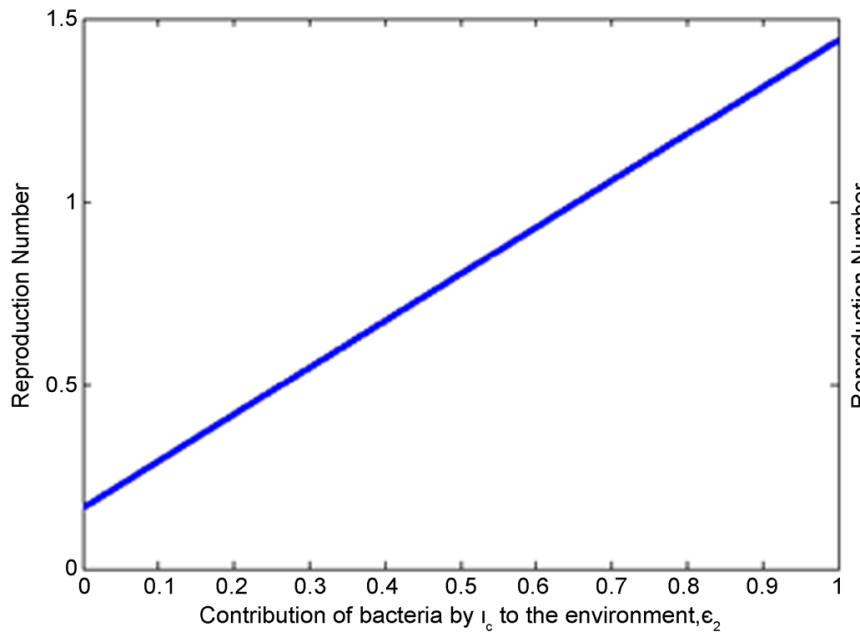

(a)

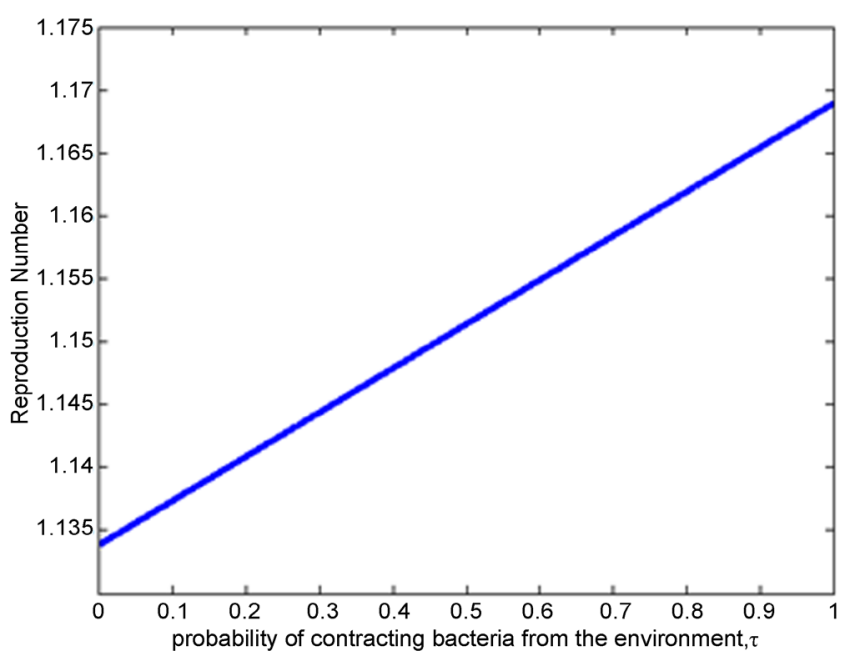

(c)

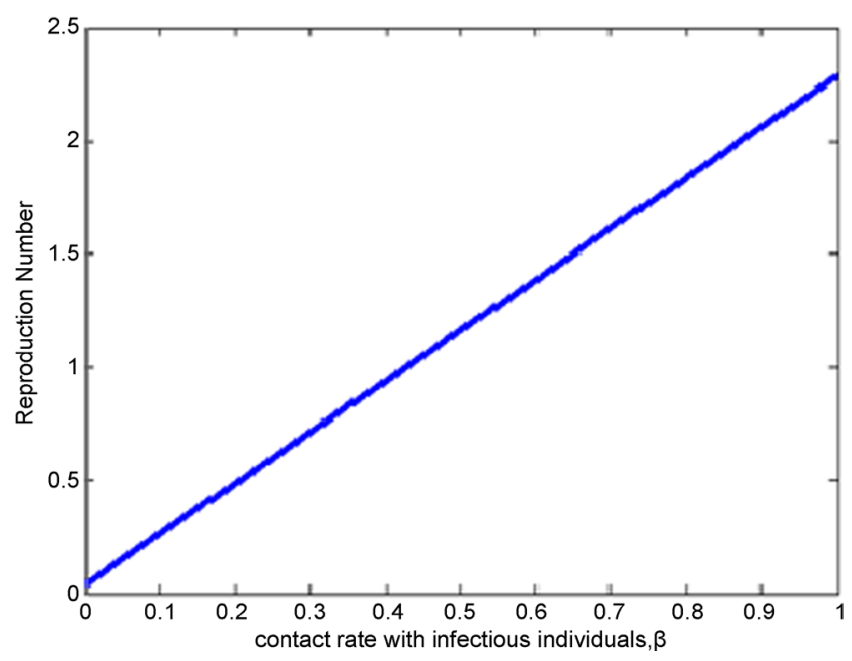

(e)

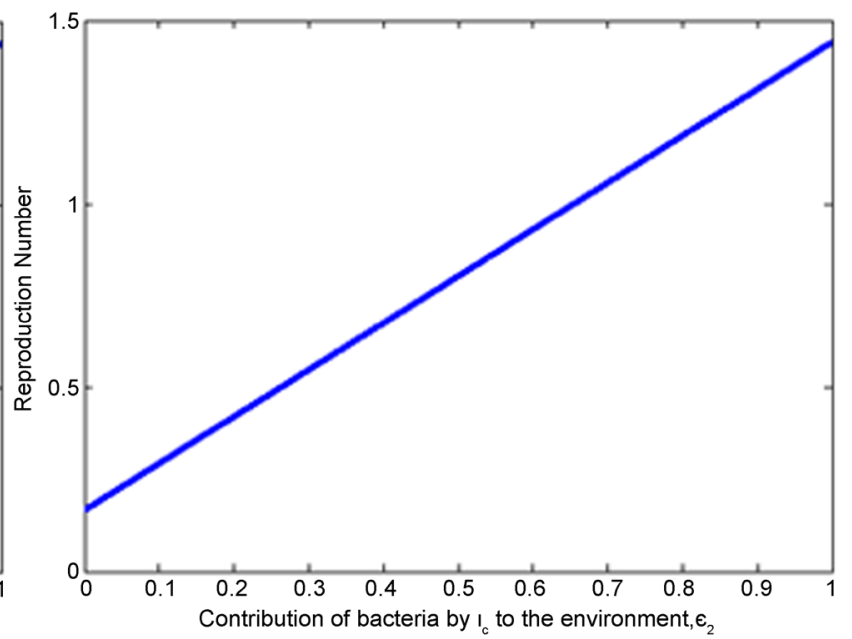

(b)

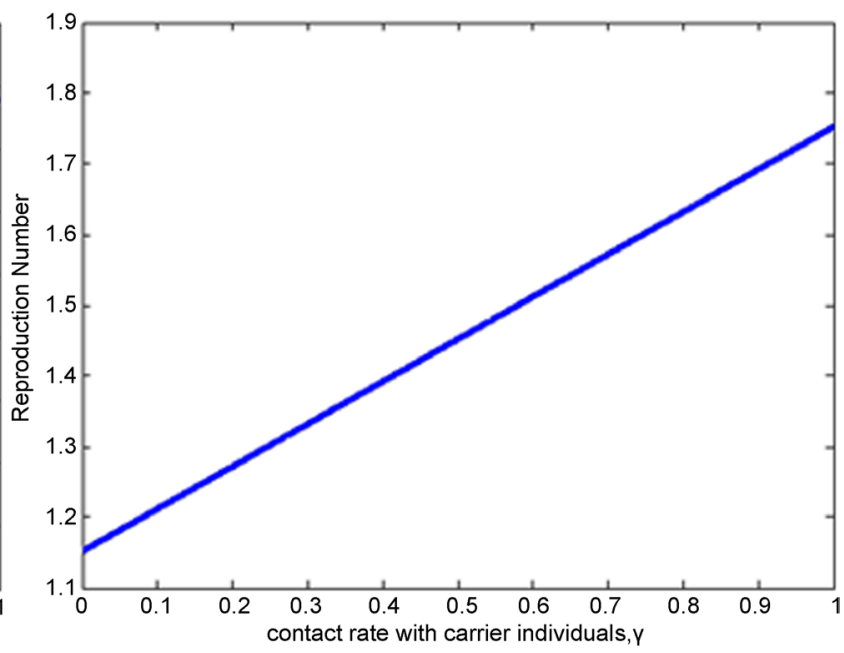

(d)

Figure 3. (a) shows the effect of infectious individuals polluting the environment with salmonela bacteria to the effective reproduction number, (b) shows the effect of carriers individuals polluting the environment with salmonela bacteria to the effective reproduction number, (c) shows probability of catching bacteria from the environment, (d) shows effect of variation of contact rate with carrier individuals on the effective reproduction number, (e) shows effect of variation of contact with infectious individual on the effective reproduction number. 
of or in conjunction with vaccination [24] [25]. The multi- compartment models suggest such a reduction in effective contact rates could lead to important reduction in prevalence [3] [26] [27]. This is consistent with Briscoe's analytical model [28].

\section{Conclusion}

In this paper, a deterministic model which incorporates person-to-person contact rate and person-environment was presented and analyzed. Important mathematical features of the models such as the threshold for the epidemic, steady states, positivity and boundedness of solutions as well as the region of biological significance were determined. The model was shown to have a disease free equilibrium which is both locally and globally asymptotically stable when the reproduction number is less than unity. This disease free equilibrium is unstable when the disease threshold is greater than unity. The model has a unique endemic equilibrium for $R_{e}>1$. In general, vaccination coverage, therapeutic treatment and water sanitation have been shown to play a pivotal role of diminishing the outbreak when they are encouraged in the community. On the other hand, it has been found beneficial to minimize contact with typhoid patients, avoid spoiling water sources with feces, and people should use Latrines. We acknowledge the fact that this work may have shortfalls as follows. The model does not take into account education campaigns. However, education is recommended as a preventive measure for typhoid. Proposed improvements of the model include consideration of a combination of hygiene, vaccination, education campaigns and biological control with salmonella specific bacteriophage.

\section{Acknowledgements}

The author would like to thank the editors of this journal for their inputs which has helped to improve this paper.

\section{References}

[1] Mushayabasa, S., Bhunu, C.P. and Ngarakana-Gwasira, E.T. (2013) Mathematical Analysis of a Typhoid Model with Carriers, Direct and Indirect Disease Transmission. International Journal of Mathematical Sciences and Engineering Applications, 7, 79-90.

[2] Mushayabasa, S. (2012) A Simple Epidemiological Model for Typhoid with Saturated Incidence Rate and Treatment Effect. International Journal of Biological, Veterinary, Agricultural and Food Engineering, 6, 56-63.

[3] Pitzer, V.E., Cayley, C., Bowles, C.C., Baker, S., Kang, G., Balaji, V., Farrar, J.J., Bryan, T. and Grenfell, B.T. (2014) Predicting the Impact of Vaccination on the Transmission Dynamics of Typhoid in South Asia: A Mathematical Modeling Study. PLOS Neglected Tropical Diseases, 8, e2642.

https://doi.org/10.1371/journal.pntd.0002642

[4] Buckle, G., Walker, C. and Black, R. (2012) Typhoid Fever and Paratyphoid Fever: Systematic Review to Estimate Global Morbidity and Mortality for 2010.Journal of Global Health, 2, 1-9. https://doi.org/10.7189/jogh.01.010401

[5] WHO (2003) Background Document, Typhoid Fever and Paratyphoid Fever. World 
Health Organization, 1-26.

[6] Adetunde, I.A. (2008) A Mathematical Models for the Dynamics of Typhoid Fever in Kassena-Nankana District of Upper East Region of Ghana. Journal of Modern Mathematics and Statistics, 2, 45-49.

[7] Kalajdzievska, D. and Li, M.Y. (2011) Modeling the Effects of Carriers on Transmission Dynamics of Infectious Disease. Mathematical Biosciences and Engineering, 8, 711-722. https://doi.org/10.3934/mbe.2011.8.711

[8] Khan, M.A., Parvez, M., Islam, S., Khan, I., Shafie, S. and Gul, T. (2015) Mathematical Analysis of Typhoid Model with Saturated Incidence Rate. Advanced Studies in Biology, 7, 65-78. https://doi.org/10.12988/asb.2015.41059

[9] Mushayabasa, S. (2014) Modeling the Impact of Optimal Screening on Typhoid Dynamics. International Journal of Dynamics and Control, 4, 330-338.

[10] Mushayabasa, S. (2011) Impact of Vaccines on Controlling Typhoid Fever in Kassena-Nankana District of Upper East Region of Ghana: Insights from a Mathematical Model. Journal of Modern Mathematics and Statistics, 5, 54-59. https://doi.org/10.3923/jmmstat.2011.54.59

[11] Edward, S. and Nyerere, N. (2016) Modeling Typhoid Fever with Vaccination and Treatment. Engineering Mathematics, 1, 44-52.

[12] Edward, S. (2017) Modeling and Stability Analysis of Typhoid Fever Transmission Dynamics with control Strategies. International Journal of Sciences. Basic and Applied Research, 32, 151-168.

[13] Baker, S., Holt, K.E., Clements, A.C.A., Karkey, A., Arjyal, A., Boni, M.F., Dongol, S., Hammond, N., Koirala, S., Duy, P.T., Nga, T.V., Campbell, J.I., Dolecek, C., Basnyat, B., Dougan, G. and Farrar, J.J. (2011) Combined High-Resolution Genotyping and Geospatial Analysis Reveals Modes of Endemic Urban Typhoid Fever, Transmission. Open Biology, 1, Article ID: 110008. https://doi.org/10.1098/rsob.110008

[14] Uneke, C.J. (2008) Concurrent Malaria and Typhoid Fever in the Tropics: The Diagnostic Challenge and Public Health Implications. Journal of Vector Borne Diseases, 45,133-142.

http://who.int/malaria/publications/atoz/9789241547925/en/index.html

[15] Mutua, J.M., Wang, F.B. and Vaidya, N.K. (2015) Modeling Malaria and Typhoid Fever Co-Infection Dynamics. Mathematical Biosciences, 264,128-144.

[16] Fujikawa, H., Kai, A. and Morozumi, S. (2004) A New Logistic Model for Escherichia Coli Growth at Constant and Dynamic Temperatures. Food Microbiology, 21, 501-509.

[17] Juneja, V.K., Melendres, M.V., Huang, L., Subbiah, J. and Thippareddi, H. (2009) Mathematical Modeling of Growth of Salmonella in Raw Ground Beef under Isothermal Conditions from 10 to 45 C. International Journal of Food Microbiology, 131, 106-111.

[18] Naresh, R. and Pandey, S. (2012) Modeling the Effect of Environmental Factors on the Spread of Bacterial Disease in an Economically Structured Population. Application and Applied Mathematics, an International Journal, 7, 426-454.

[19] Pearl, R. (1927) The Growth of Populations. The Quarterly Review of Biology, 2, 532-548.https://doi.org/10.1086/394288

[20] Vadasz, A.S., Vadasz, P., Abashar, M.E. and Gupthar, A.S. (2001) Recovery of an Oscillatory Mode of Batch Yeast Growth in Water for a Pure Culture. International Journal of Food Microbiology, 71, 219-234.

[21] Verhulst, P.F. (1838) Notice surla loique la population suit dansson accrois-sement. 
Corr.Math. et Phys.Publ. par A. Quetelet. TX, 113-121.

[22] Diekmann, O., Heesterbeek, J.A.P. and Roberts, M.G. (2009)The Construction of Next-Generation Matrices for Compartmental Epidemic Models. Journal of the Royal Society Interface, 7, 873-885.

[23] Van den Driessche, P. and Watmough, J. (2002) Reproduction Numbers and Sub-Threshold Endemic Equilibria for Compartmental Models of Disease Transmission. Mathematical Biosciences, 180, 29-48.

[24] Velema, J.P., van Wijnen, G., Bult, P., van Naerssen, T. and Jota, S. (1997) Typhoid Fever in Ujung Pandang, Indonesia? High-Risk Groups and High-Risk Behaviors. Tropical Medicine \& International Health, 2, 1088-1094. https://doi.org/10.1046/j.1365-3156.1997.d01-179.x

[25] Greenwell, J., McCool, J., Kool, J. and Salusalu, M. (2012) Typhoid Fever: Hurdles to Adequate Hand Washing for Disease Prevention among the Population of a Peri-Urban Informal Settlement in Fiji. Western Pacific Surveillance and Response Journal, 3, 41-45.

[26] Gonzalez-Guzman, J. (1989) An Epidemiological Model for Direct and Indirect Transmission of Typhoid Fever. Mathematical Biosciences, 96, 33-46.

[27] Bailey, N.J. (1982) The Structural Simplification of an Epidemiological Compartment Model. Journal of Mathematical Biology, 14,101-116.

[28] Briscoe, J. (1980) On the Use of Simple Analytic Mathematical Models of Communicable Diseases. International Journal of Epidemiology, 9, 265-270.

Submit or recommend next manuscript to OALib Journal and we will provide best service for you:

- Publication frequency: Monthly

- 9 subject areas of science, technology and medicine

- Fair and rigorous peer-review system

- Fast publication process

- Article promotion in various social networking sites (LinkedIn, Facebook, Twitter, etc.)

- Maximum dissemination of your research work

Submit Your Paper Online: Click Here to Submit

Or Contact service@oalib.com 